Research Papers

\title{
Effects of Substrate Temperature on Properties of Tin-Doped Indium Oxide Films Deposited by Activated Electron Beam Evaporation
}

\author{
Hiroyasu KOJIMA ${ }^{\mathrm{a}, *}$, Nagahiro SAITO ${ }^{\mathrm{b}}$ and Osamu TAKAI ${ }^{\mathrm{b}}$ \\ ${ }^{\text {a }}$ Earth-tech Ltd. (4-5-21, Kokubu-kita, Ebina-shi, Kanagawa 243-0406) \\ ${ }^{\mathrm{b}}$ Graduate School of Engineering, Nagoya University (Furo-cho, Chikusa-ku, Nagoya-shi, Aichi 464-8603)
}

\begin{abstract}
The coating process was conducted using an inline-type plasma deposition system. Thin and thick films containing $\mathrm{SnO}_{2}$ were prepared from the starting material sintered ITO pellets $\left(0-15 \mathrm{wt} \% \mathrm{SnO}_{2}\right)$. The thin film $(<0.6 \mu \mathrm{m}$ thick $)$ showed an amorphous-like structure. The thick film ( $>1 \mu \mathrm{m}$ thick) showed a well-ordered crystalline structure. The crystalline and electrical properties of the films were dependent on the deposition temperature and were closely related to the $\mathrm{SnO}_{2}$ contents. Films deposited on substrates at temperatures of $50-180^{\circ} \mathrm{C}$ comprised a mixture of amorphous and polycrystalline phases and had a hazy appearance because of light scattering at the rough surface. That appearance was not observed in films with $0 \mathrm{wt} \% \mathrm{SnO}_{2}$ content, but it was observed with increasing $\mathrm{SnO}_{2}$ content and substrate temperature. In films with thickness greater than $0.6 \mu \mathrm{m}$, the hazy appearance was more pronounced than in films with thickness of less than $0.6 \mu$ $\mathrm{m}$. Polycrystalline ITO films with a clean and transparent appearance, as well as minimum resistivity of $1.7 \times 10^{-4} \Omega \mathrm{cm}$, were obtained on substrates at $180{ }^{\circ} \mathrm{C}\left(4 \mathrm{wt} \%, 7.5 \mathrm{wt} \% \mathrm{SnO}_{2}\right.$ ). Transparent and uniform ITO films with sheet resistance of $1.7 \Omega / \square$ were obtained at $2.1 \mu \mathrm{m}$ thickness at a high deposition rate (ca. $0.5 \mu \mathrm{m} / \mathrm{min}$ ).
\end{abstract}

Keywords : Substrate Temperature, Low Resistance, ITO Film, Hazy Appearance

\section{Introduction}

Electrically conductive, transparent $\mathrm{Sn}$-doped $\mathrm{In}_{2} \mathrm{O}_{3}$ (ITO) films are widely used in various fields. Electron beam (EB) evaporation and sputtering are the main technologies currently used to produce these films. However, it is difficult to obtain low-resistance (1-10 $\Omega / \square)$ ITO films on large-scale substrates at low substrate temperatures using these methods, for example, for architectural or automotive windows.

To date, various ion- and plasma-based technologies have been studied with the aims of reducing the resistivity, increasing the deposition rate, and reducing the substrate temperature during deposition. These methods include electron enhanced ion plating (EEIP $)^{1-2)}$, high-density plasma-assisted evaporation $(\mathrm{HDPE})^{3)-6)}$, and low voltage dc magnetron sputtering at a decreased plasma impedance. ${ }^{7)}$

As for the production of large area ITO, the low voltage sputtering method is currently the main process used, ${ }^{7)}$ but because the substrate used has changed from glass to plastic films, deposition at low temperatures with low damage has become necessary. In the sputtering method, the increase in substrate temperature at low deposition rates and the damage to the substrate caused by the high-energy sputter particles remain problematic.

Recently, because of the high costs due to the scarcity of indium oxide, alternative transparent and electroconductive materials have been actively studied. Yamamoto et al. ${ }^{8}$ reported a transparent conductive film of a $\mathrm{ZnO}$ material fabricated using the HDPE method, which is the same method as used in this work. The $\mathrm{ZnO}$ film has problems of durability and poor etching characteristics, and if the $\mathrm{ZnO}$ material is not mass-produced, the costs are high. Ichinose et al. ${ }^{9)}$ described a development method using $\mathrm{TiO}_{2}$ material and the pulse laser deposition (PLD) method, but it is difficult to fabricate a large-area product in this method. In addition, $\mathrm{TiO}_{2}$ is durable and lithography is therefore difficult.

As for ITO films, the collection and recycling of the used target material is widely practiced, and efforts are being made toward the effective use of resources. In addition, the applications of touch panels and so on are increasing rapidly, but there is very little consumption of the material in the films of such products. In the future, various materials that are most suited for particular applications will be used, and more detailed research on ITO films will be necessary.

At the same time, for deposition at low temperatures, the use of plasma is important for improving the thin-film properties. This process, using low electron temperatures and high electron densities, has great appeal as a plasma source. In order to achieve our aim, we studied high-density plasma-assisted EB evaporation (HDPE). The electron beam and plasma were produced by a plasma-generator system based on the arc-discharge process. The plasma gun was used in a dc hollow cathode discharge with a pressure gradient. The plasma gun has a cathode system composed of $\mathrm{a} \mathrm{LaB}_{6}$ disc and a tantalum pipe. The essential feature of this gun is its low-voltage, high-current electron beam. The plasma density of the arc-discharge-type plasma obtained from such a system is two or three orders of magnitude higher than that of the glow-discharge-type plasma used in conventional magnetron sputtering and ion plating. Therefore, one can expect a high ionization ratio and a large quantity of activated neutral species from this process. This report describes the dependences of the structural, electrical, and optical properties of ITO films prepared by HDPE on the process parameters of EB current, oxygen flow rate, and substrate temperature and thickness.

\footnotetext{
*E-mail: earth-tech@r9.dion.ne.jp
} 


\section{Experimental procedures}

\section{1 Coater}

The deposition technique used here is low-voltage highcurrent electron beam (EB) deposition (Fig. 1). The distance between source and substrate is $0.7 \mathrm{~m}$. Thermally emitted electrons from the cathode surface were focused on the sintered ITO pellets by guiding them to the hearth (anode) by applying a magnetic field. Arc discharge was maintained between the cathode and the anode with the aid of discharge argon gas introduced into the cathode region. All coatings in these experiments were carried out at a discharge current of 150-300 A, discharge voltage of $70 \mathrm{~V}$. Fig. 2 shows a schematic diagram of the in-line-type coater with HDPE system that was used. This coater has a lock chamber with a heating system, a deposition chamber with two sets of HDPE guns, and an overrun chamber.

\section{2 Deposition Procedure}

ITO sintered pellets $\left(\mathrm{In}_{2} \mathrm{O}_{3}+0-15 \mathrm{wt} \% \mathrm{SnO}_{2}\right)$ were used as the starting materials. Float glass plates sized $1 \times 1 \mathrm{~m}$ were used as the substrates. The glass substrates is preheated at each temperature from the room temperature to $200{ }^{\circ} \mathrm{C}$ at the loadlock chamber and it carried them to the evaporation chamber. The whole system was first evacuated to a vacuum of $\sim 10^{-4}$ Pa using a conventional oil-diffusion pump system. Then, argon as the discharge gas and oxygen as the reactive gas were intro-

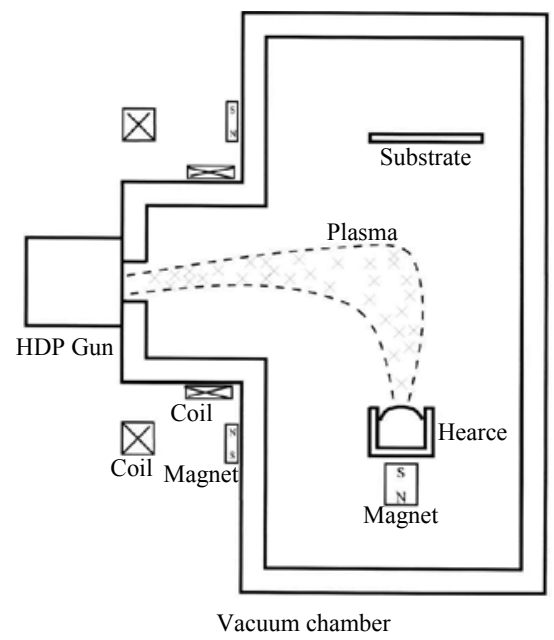

Fig. 1 Schematic diagram of highly dense plasmaassisted EB evaporation (HDPE) system.

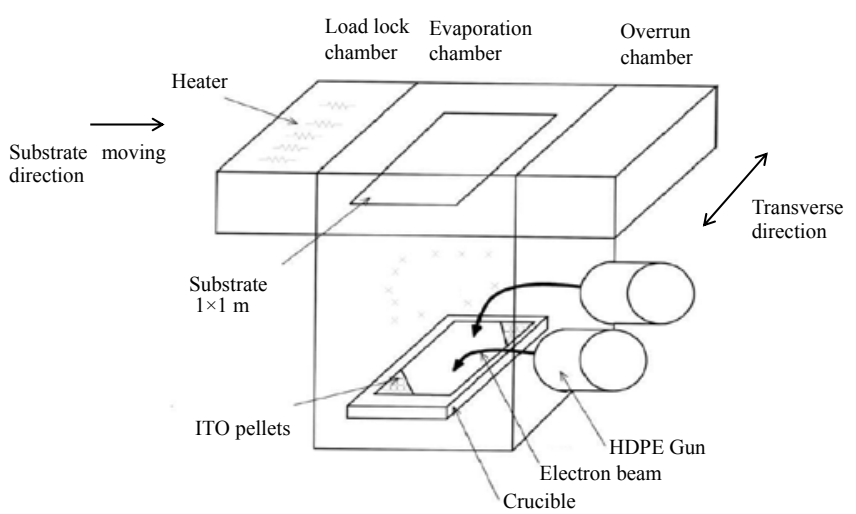

Fig. 2 Schematic diagram of experiment apparatus duced into the deposition chamber. The total gas pressure was controlled at about $2 \times 10^{-1} \mathrm{~Pa}$ by means of mass flow controllers. A low energy electron beam accompanied by highly dense plasma was used to irradiate the ITO pellets through the evaporating zone.

\section{3 Evaluation Techniques}

Film thickness was measured using a stylus-type thickness monitor (Sloan, Dektak3) Sheet resistance, resistivity by a conventional four-probe arrangement (Napson, RT-70), Hall mobility, and carrier density were measured by Hall effect measurements in the van der Pauw method (BIORAD, HL5500PC). Optical transmission were measured in the wavelength range 350-850 nm using spectrometer (Hitachi, U-4000). Visible transmission (\%) used transmittance at the $550 \mathrm{~nm}$ wavelength. The structural characteristics of the films were studied by X-ray diffraction analysis with $55 \mathrm{kV}, 200 \mathrm{~mA} \mathrm{CuK} \alpha$ radiation (Rigaku, RU-200RADB). Surface morphology was observed using scanning electron microscopy (Hitachi, S-900).

\section{Results and Discussion}

\section{1 Deposition Rate}

Fig. 3 shows deposition rate, resistivity, and visible transmission $\left(\mathrm{T}_{\mathrm{v}}\right)$ of the ITO films deposited by HDPE as functions of electron beam current introduced from the generators to the ITO source. Both the deposition rate and $\mathrm{T}_{\mathrm{v}}$ increased with an increase in EB current. In conventional EB evaporation, $T_{v}$ decreases with an increase in the evaporation rate because a non-stoichiometric film forms when the oxygen flow rate is constant. ${ }^{10)}$ The increase in $T_{v}$ seen in Fig. 3 is a feature of the present process, which is due to an increase in the plasma density above the source with an increase in the EB current. Therefore, oxidation was enhanced even if the flux density of the ITO vapor increased. The resistivity exhibited a minimum value at $300 \mathrm{~A}$, and this resistivity was reduced further by optimizing the oxygen gas flow rate.

Table 1 shows the dependency of resistivity on oxygen flow rate. The substrate was preheated to $70{ }^{\circ} \mathrm{C}$. The resistivity

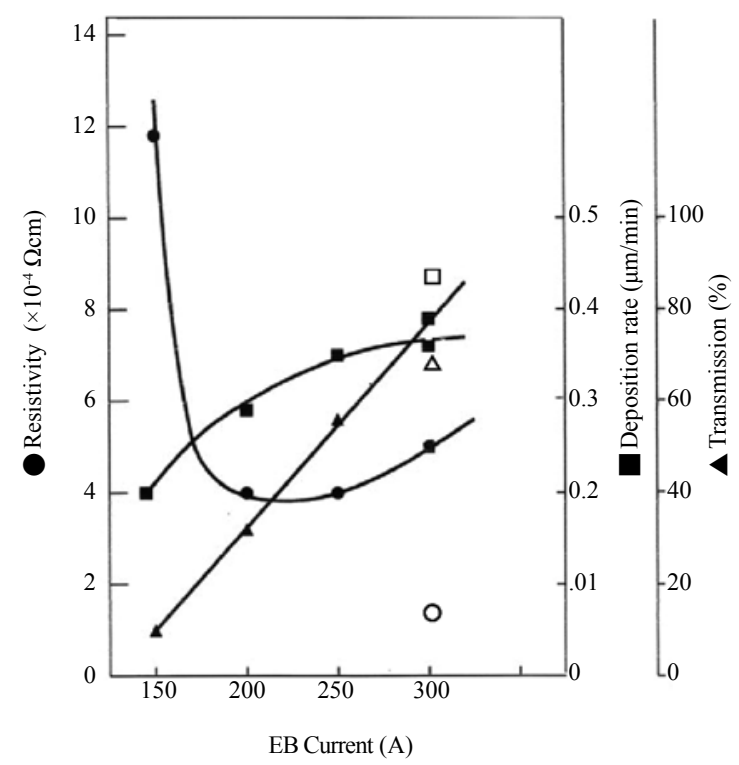

Fig. 3 Resistivity, transmission and deposition rate versus EB current.

AD Flow rate $\mathrm{O}_{2}: 65$, Ar: $50 \mathrm{sccm}$ Flow rate $\mathrm{O}_{2}: 65$, Ar: $35 \mathrm{sccm}$ $\left(\mathrm{SnO}_{2}\right.$ content: $\left.4.0 \mathrm{wt} \%\right)$ 
gradually decreased from $6.4 \times 10^{-4}$ to $2.8 \times 10^{-4} \Omega \mathrm{cm}$ with a decrease in oxygen flow rate. This decrease was due to a variation of the carrier concentrations.

As shown in Fig. 3, a deposition rate of $0.5 \mu \mathrm{m} / \mathrm{min}$. was obtained with an EB current of 300 A by optimizing the discharge gas flow rate. This deposition rate is about ten-times higher than that of conventional thermal evaporation methods. ${ }^{10)}$ In this process, ITO pellets were heated by a high-current electron beam accompanied by argon plasma, and were evaporated at a high evaporation rate. A large number of ions and activated neutral atoms of indium and oxygen were produced in the highly dense argon plasma above the evaporation crucible. Furthermore, a glass substrate in contact with the plasma had a negative selfbias potential of $10-20 \mathrm{~V}$, which acted as an acceleration voltage in the ion plating process. The reactivity of oxygen to indium and crystallization of the ITO films were enhanced and high quality ITO films were prepared at low substrate temperature and at high deposition rate.

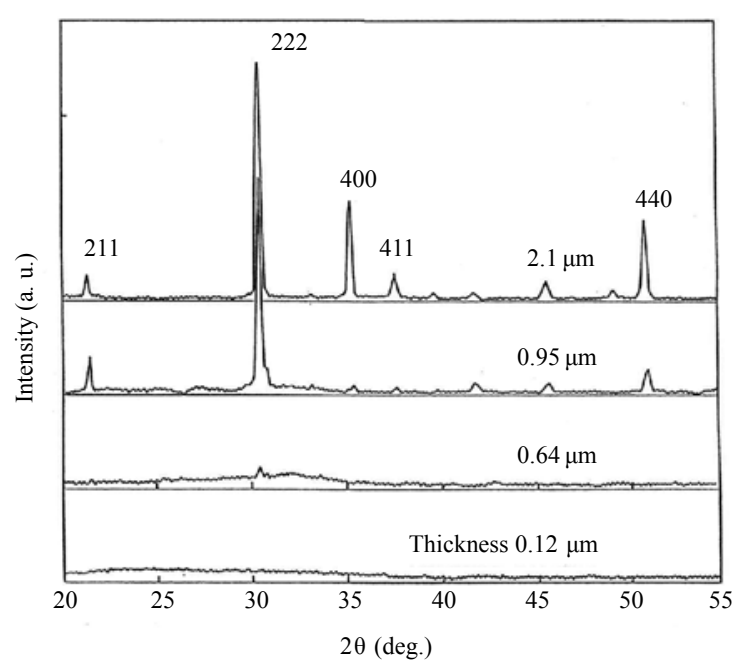

Fig. 4 X-ray diffraction patterns of the ITO films with different thickness. (Without heating a substrate, $\mathrm{SnO}_{2}$ content: $7.5 \mathrm{wt} \%$ )

\section{2 Thickness dependency}

Fig. 4 shows the XRD of the ITO films with various thicknesses. The films with a thickness of less than $0.6 \mu \mathrm{m}$ exhibited amorphous-like diffraction patterns.

In the case of the $1-\mu \mathrm{m}$-thick films, peaks corresponding to the (222) plane of $\operatorname{In}_{2} \mathrm{O}_{3}$ emerged in the amorphous diffraction patterns. This crystallization was due to an increase in the substrate temperature. Since the glass surface was exposed to dense plasma, the films were heated gradually with an increase in thickness and resulted in crystallization with (222) preferred orientation.

Table 2 shows the thickness dependency of the films electrical properties and $\mathrm{T}_{\mathrm{v}}$. Thicker films showed lower resistance and resistivity. For example, the film of $2.1 \mu \mathrm{m}$ thickness had a sheet resistance of $1.7 \Omega / \square$ and $\mathrm{T}_{\mathrm{v}}$ of about $61 \%$. The transparency was improved by optimizing the oxygen flow rate.

\section{3 Effects of substrate temperature}

The three films marked A, B, and C in Fig. 5 were investigated

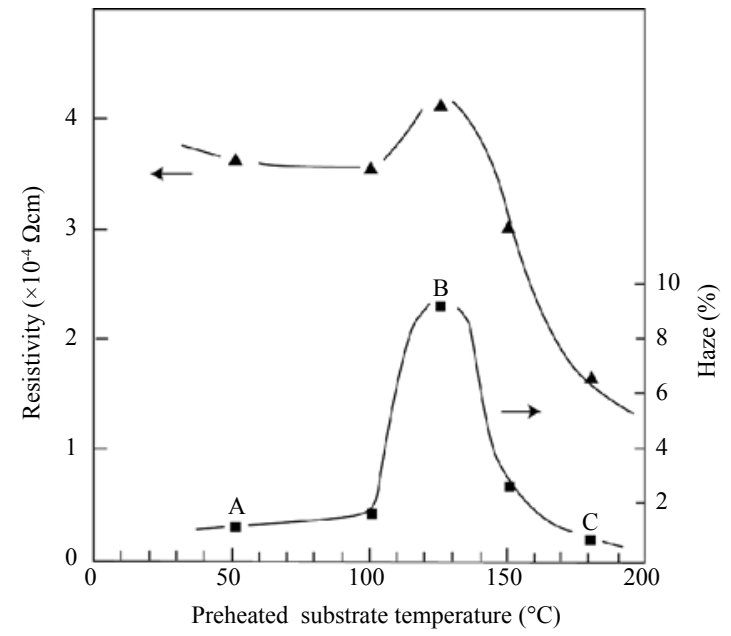

Fig. 5 Dependence of resistivity and haze of ITO films on the substrate temperature.

$\left(\mathrm{SnO}_{2}\right.$ content: $\left.7.5 \mathrm{wt} \%\right)$

Table 1 Properties of ITO films deposited at different $\mathrm{O}_{2}$ gas flow rates.

\begin{tabular}{|c|c|c|c|c|c|c|}
\hline $\begin{array}{c}\mathrm{O}_{2} \text { gas flow rate } \\
(\mathrm{sccm})\end{array}$ & $\begin{array}{l}\text { Thickness } \\
\qquad(\mu \mathrm{m})\end{array}$ & $\begin{array}{c}\text { Resistivity } \\
\left(\times 10^{-4} \Omega \mathrm{cm}\right)\end{array}$ & $\begin{array}{l}\text { Carrier concentration } \\
\qquad\left(\times 10^{20} \mathrm{~cm}^{-3}\right)\end{array}$ & $\begin{array}{l}\text { Hall mobility } \\
\left(\mathrm{cm}^{2} \mathrm{~V}^{-1} \mathrm{~s}^{-1}\right)\end{array}$ & Prefered orientation & $\begin{array}{c}\text { Visible transmission } \\
(\%)\end{array}$ \\
\hline 60 & 1.12 & 6.4 & 2.9 & 34 & (111) & 76.2 \\
\hline 50 & 0.955 & 6.1 & 2.0 & 51 & (111) & 79.1 \\
\hline 40 & 1.06 & 3.7 & 5.5 & 30 & (111) & 77.2 \\
\hline 30 & 1.01 & 2.8 & 5.1 & 44 & (111) & 76.1 \\
\hline below 10 & \multicolumn{6}{|l|}{ Absorbing film } \\
\hline
\end{tabular}

Preheating of the substrate : $70{ }^{\circ} \mathrm{C}, \mathrm{EB}$ current : $250 \mathrm{~A}$, voltage : $70 \mathrm{~V}$

Table 2 Properties of ITO films with different thicknesses.

\begin{tabular}{c|c|c|c|c|c}
\hline $\begin{array}{c}\text { Thickness } \\
(\mu \mathrm{m})\end{array}$ & $\begin{array}{c}\text { Sheet resistance } \\
(\Omega / \square)\end{array}$ & $\begin{array}{c}\text { Resistivity } \\
\left(\times 10^{-4} \Omega \mathrm{cm}\right)\end{array}$ & $\begin{array}{c}\text { Carrier concentration } \\
\left(\times 10^{20} \mathrm{~cm}^{-3}\right)\end{array}$ & $\begin{array}{c}\text { Hall mobility } \\
\left(\mathrm{cm}^{2} \mathrm{~V}^{-1} \mathrm{~s}^{-1}\right)\end{array}$ & $\begin{array}{c}\text { Visible transmission } \\
(\%)\end{array}$ \\
\hline 0.12 & 61 & 7.4 & 2.2 & 38 & 82.0 \\
0.64 & 7.8 & 5.0 & 2.4 & 53 & 79.1 \\
0.95 & 4.4 & 4.2 & 4.1 & 36 & 75.2 \\
2.15 & 1.7 & 3.6 & 7.4 & 23 & 61.2 \\
\hline
\end{tabular}

Without preheating a substrate, Oxygen flow rate of $40 \mathrm{sccm}, \mathrm{SnO}_{2}$ content : $7.5 \mathrm{wt} \%$ 
using XRD. Fig. 6 shows the results of this XRD analysis. Film A, deposited without preheating was in an amorphous phase and film C, deposited on a substrate preheated at $180{ }^{\circ} \mathrm{C}$ was polycrystalline. Both these films exhibited a low haze value as shown in Fig. 5. Film B, deposited on a substrate preheated at $125{ }^{\circ} \mathrm{C}$ was composed of coexisting amorphous and crystalline phases, which is clearly demonstrated by the halo pattern around the (222) peak of $\operatorname{In}_{2} \mathrm{O}_{3}$ in the XRD pattern. In the initial stage of the deposition, the substrate temperature (around $125^{\circ} \mathrm{C}$ ) is just below the crystallization temperature of ITO. The film begins to grow in the amorphous phase. During the process, the substrate temperature gradually increases because of the high radiation produced by the evaporation sources. Additionally, Langmuir probe measurements showed that the plasma density near the substrate was of the order of $10^{13}$ ions $\mathrm{cm}^{-3}$, which is extremely high compared with densities observed in other processes based on glow discharge. When the substrate temperature reaches the crystallization temperature, deposited atoms and molecules form the crystalline ITO film on top of the amorphous film. At almost the same time, the underlying amorphous layer begin to recrystallize. Fig. 7 shows an SEM image of a cross-section of the film B in Fig. 5.

\section{4 Effects of $\mathrm{SnO}_{2}$ contents}

It is clear that a hazy appearance and crystallization are closely related. We changed the substrate temperature to determine the optimum content of $\mathrm{SnO}_{2}$ to give the lowest resistivity without a hazy appearance (Fig. 8 a-d).

From the results in previous reports ${ }^{3)-5)}$, it was found that in the process of recrystallization, the volume of the film decreases because of the difference in density between the two phases. The surface profiles of the films showed a smooth surface and a surface with small irregularities that are considered to correspond to individual crystal grains. The roughness formed on the surface of the film seems to scatter the light, and it appears clouded; this is called "haze", and with this haze, the transmittance decreases.

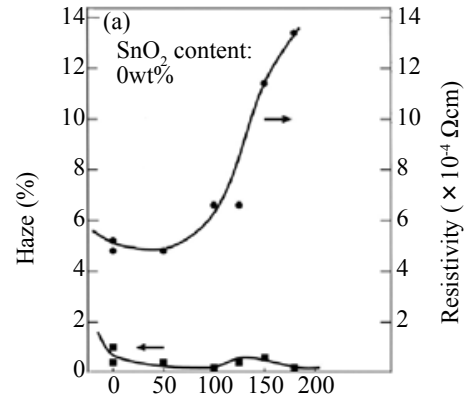

Preheated substrate temperature $\left({ }^{\circ} \mathrm{C}\right)$

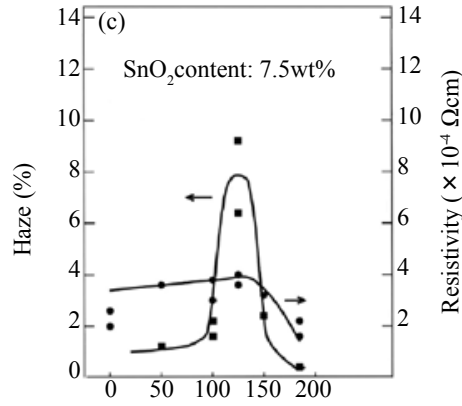

Preheated substrate temperature $\left({ }^{\circ} \mathrm{C}\right)$

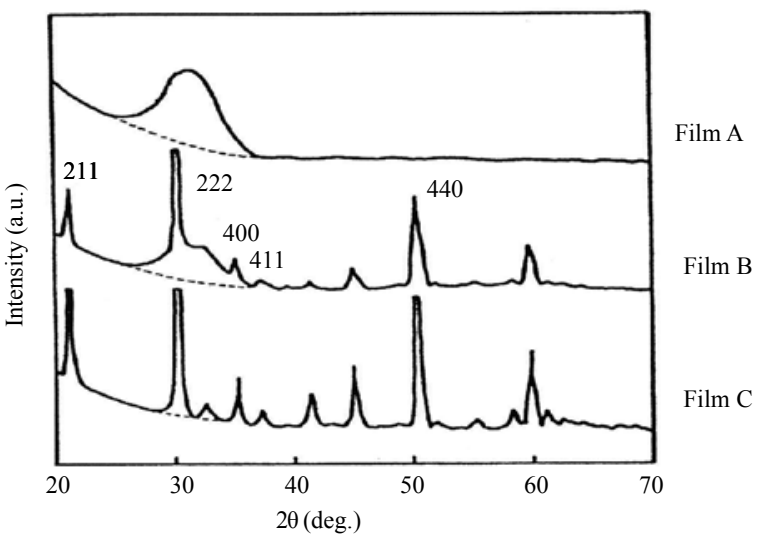

Fig. 6 X-ray diffraction patterns of ITO films deposited at different the temperatures.

Film A: $50{ }^{\circ} \mathrm{C}$, film B: $125^{\circ} \mathrm{C}$, film C: $180{ }^{\circ} \mathrm{C}$.

(Thickness: $1 \mu \mathrm{m}, \mathrm{SnO}_{2}$ content: $7.5 \mathrm{wt} \%$ )

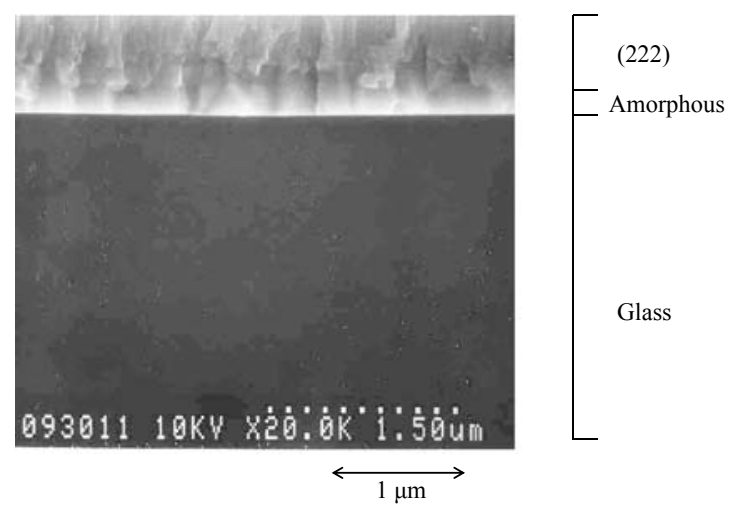

Fig. 7 SEM photograph of the cross section of $0.7 \mu \mathrm{m}$ thick ITO film deposited at the preheating temperature of $125^{\circ} \mathrm{C}$. $\left(\mathrm{SnO}_{2}\right.$ content: $\left.7.5 \mathrm{wt} \%\right)$

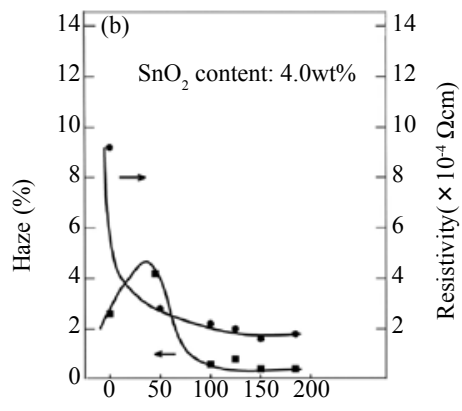

Preheated substrate temperature $\left({ }^{\circ} \mathrm{C}\right)$

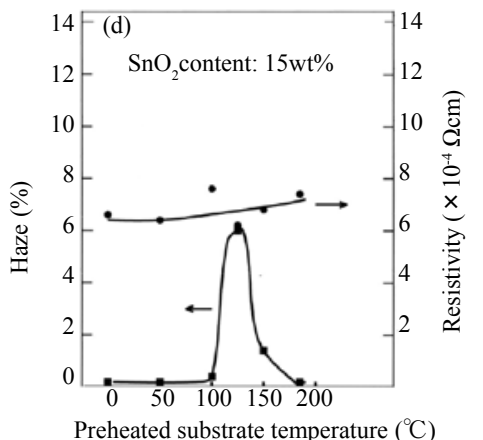

Fig. 8 Haze and electrical properties of ITO films deposited at different substrate temperatures. 
Also it was found when the amount of $\mathrm{SnO}_{2}$ in the ITO film is increased, the temperature of the crystallization increases ${ }^{11)}$.

In this study, we examined haze formation for different $\mathrm{SnO}_{2}$ content. Haze does not occur in the monophase, as in amorphous or crystal phase. For haze to occur, the amorphous phase must crystallize during the deposition process. With a low $\mathrm{SnO}_{2}$ content, the crystallization of the ITO film progresses at a low preheated substrate temperature. Because the crystallization began with a $\mathrm{SnO}_{2}$ content of $4 \mathrm{wt} \%$ without substrate preheating, the haze increased with the heating temperature, and the crystallization was complete at a preheated substrate temperature of 100 ${ }^{\circ} \mathrm{C}$, haze is decreased (Fig. 8 b). With $\mathrm{SnO}_{2}$ content of $7.5 \mathrm{wt} \%$ and $15 \mathrm{wt} \%$, the crystallization temperature of the ITO film rises, and the maximum haze value occurs at a preheated substrate temperature of $125^{\circ} \mathrm{C}$. It is thought that the substrate temperature rises during deposition, so the crystallization temperature is actually higher than $125{ }^{\circ} \mathrm{C}$. Because the crystallization temperature increases, the volume of the amorphous film also increases with film thickness. Therefore, the initially decreasing volume of the crystallized amorphous film increases, along with the haze value. In addition, it is thought that the crystallization of the ITO film is complete at a preheated substrate temperature of $150{ }^{\circ} \mathrm{C}$ (Fig. $8 \mathrm{c}$, d). With a $\mathrm{SnO}_{2}$ content of $7.5 \mathrm{wt} \%$, it is thought that the crystallization of the ITO film begins at a preheated substrate temperature of $100{ }^{\circ} \mathrm{C}$ or less $($ Fig. 8 c). Fig. 9 shows the relationship between the haze and the film thickness. It was found that the haze value of the film increases with film thickness. A minimum thickness is necessary for crystallization from the amorphous phase. The hazy appearance is more pronounced in films with thicknesses greater than $0.6 \mu \mathrm{m}$ than in thinner films.

Fig. 4 shows that the preferred orientation changes from (222) to (400) with increasing film thickness. Shigesato et al. compared the preferred orientations of the sputtered film, the EB film, and the ITO powder. ${ }^{6}$ The peak intensity ratios of $(400) /(222)$ were 11.6, 0.14, and 0.37 for the sputtered film, EB film, and ITO powder, respectively. The increase in the lattice parameters to the ASTM values indicates uniform strain in the films. The values which is the increase in the lattice parameter of the sputtered films were $0.99 \%$ and $0.74 \%$ for the (222) and (400) planes, respectively. This difference should be attributed to the predominant growth of grains with $<100>$ orientation over those with

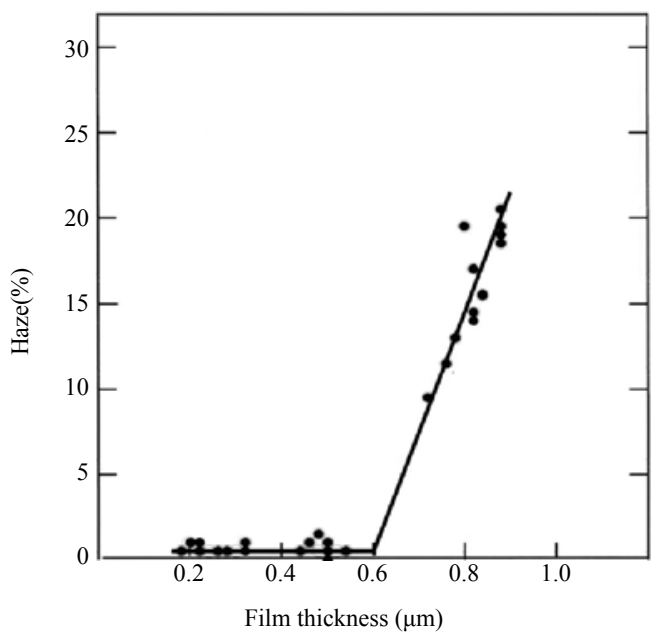

Fig. 9 Haze versus thickness of film deposited at the preheating temperature of $125{ }^{\circ} \mathrm{C} .\left(\mathrm{SnO}_{2}\right.$ content: $\left.7.5 \mathrm{wt} \%\right)$
$<111>$ orientation. That is, as the driving force, crystal growth may occur in the direction that reduces strain. It is thought that a similar mechanism works when the film thickness is increased.

\section{5 Resistivity}

On the basis of the previous results ${ }^{3), 4)}$, He was used instead of Ar as the discharge gas. The rate in He was about six times greater than that in Ar, but the discharge voltage was $30 \mathrm{~V}$ higher than that in Ar at the same current. The specific resistivity was very low, even at low temperatures. The rise in resistivity at a preheated substrate temperature of around $125{ }^{\circ} \mathrm{C}$ is considered to be related to the complex crystallization behavior around this temperature. He gas has a higher ionization potential than $\mathrm{Ar}$, and it is not easy to produce a stable high-current He discharge.

In this study, we examined the dependence of the specific resistivity in $\mathrm{Ar}$ on the substrate temperature. Fig. 10 shows this dependence for a $\mathrm{SnO}_{2}$ content of about $4 \mathrm{wt} \%$, which provides the lowest specific resistivity. The specific resistivity of the ITO film decreases rapidly with increasing preheated substrate temperature. Even if the substrate temperature is changed, the mobility remains roughly the same. The main reason for this decrease in specific resistivity is the increase in carrier concentration. Because the $\mathrm{SnO}_{2}$ content here is constant, the increase in carrier concentration is thought to correspond to the increase in oxygen vacancies. Table 1 shows the specific resistivities, carrier concentrations, and Hall mobilities for different amounts of oxygen. When the amount of oxygen changes, the carrier concentration roughly changes with it and is the dominant one among the above-mentioned properties. This is found to be an important factor in the optimization of the $\mathrm{O}_{2}$ amount for the fabrication of low-resistivity ITO films.

In section 3.4, it was shown that haze is mainly connected with the structural change upon crystallization from the amorphous phase. The increase in haze value is thought to indicate a change from the amorphous to the crystalline phase, after which the haze value decreases (Fig. 8 b). That is, the mobility does not change too much even if the substrate temperature increases and crystallization occurs (Fig. 10). The decrease in carrier mobility may originate from various scattering mechanisms. ${ }^{12)}$ In the case of ITO, the scattering can be classified as (1) ionized impurity scattering, (2) neutral impurity scattering, (3) grain-boundary scattering, or (4) lattice scattering. Of these, lattice scattering (4)

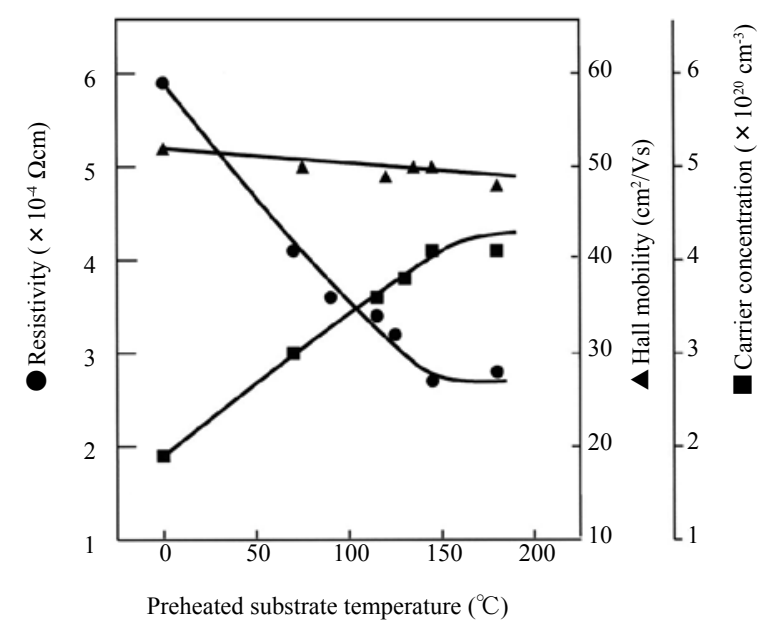

Fig. 10 Electrical properties versus substrate temperature. ( $\mathrm{O}_{2}$ flow rate: $70 \mathrm{sccm}, \mathrm{SnO}_{2}$ content: $4.0 \mathrm{wt} \%$ ) 


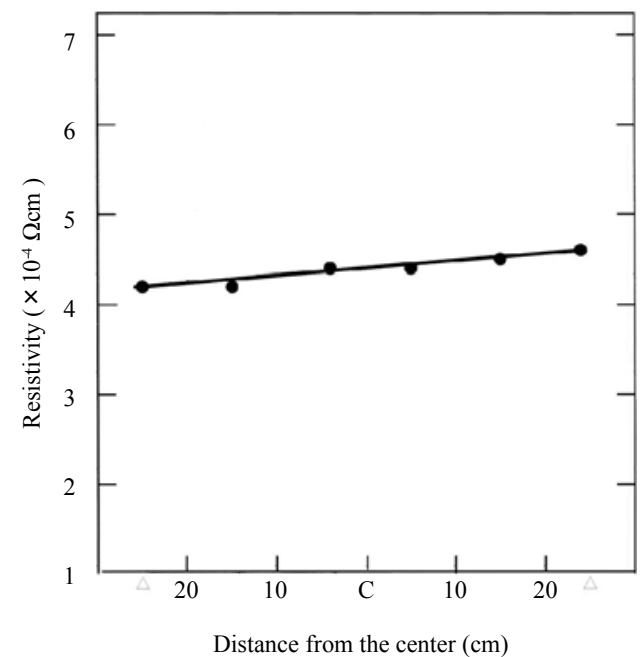

Fig. 11 Transverse distribution of the resistivity of the ITO films using a mask. $\left(\mathrm{SnO}_{2}\right.$ content: $\left.7.5 \mathrm{wt} \%\right)$

is not the major scattering mechanism at low temperatures. It is thought that grain-boundary scattering (3) can also be ignored in these results.

Table 2 shows the dependences of the carrier concentration, the Hall mobility, and the resistivity on the film thickness. The carrier concentration increases simply with increasing film thickness. It was found that the decrease in specific resistivity with increasing film thickness follows a similar mechanism to that of the substrate temperature. It was also confirmed that the carrier concentration in the sputtered film increases with increasing film thickness. ${ }^{6}{ }^{6}$ This suggests that a trap for the crystalline defects of the carrier concentration of dopants ( $\mathrm{Sn}$ or O vacancies) decreases because the crystallinity is improved.

\section{6 Uniformity}

Fig. 11 shows a transverse distribution of the resistivity of an ITO film.

The film thickness was about $1 \mu \mathrm{m}$. The film showed adequate uniformity between two source ( $0.5 \mathrm{~m}$ wide), which demonstrates the possibility of scale up by increasing the number of sources.

\section{Conclusions}

A newly developed in-line coater based on highly dense plasma assisted EB evaporation (HDPE) has been used to pre- pare low resistance ITO films on large-scale glass substrates. By optimizing process parameters, such as EB current and oxygen flow rate, ITO films with resistivities of $1.7 \times 10^{-4} \Omega \mathrm{cm}$ were obtained at a deposition rate of $0.5 \mu \mathrm{m} / \mathrm{min}$. Sheet resistances of $1.7 \Omega / \square$ were obtained by increasing the film thickness.

Thin films $(<0.6 \mu \mathrm{m})$ with an amorphous-like structure, medium-thickness films with mixed amorphous and crystalline phases, and thick films $(>1 \mu \mathrm{m})$ with only crystalline phase were produced. This crystallinity achieved was due to the increase in substrate temperature during deposition.

The haze value of the films was maximum at a preheated substrate temperature of $125{ }^{\circ} \mathrm{C}$, in $7.5 \mathrm{wt} \% \mathrm{SnO}_{2}$ contents. The ITO film deposited at $180{ }^{\circ} \mathrm{C}$ exhibited the minimum resistivity of 1.7 $\times 10^{-4} \Omega \mathrm{cm}$ and was obtained as a polycrystalline phase without haze. Therefore, it was possible to produce a film with low resistivity and without haze by using $4 \mathrm{wt} \% \mathrm{SnO}_{2}$ and a low substrate temperature.

\section{Acknowledgement}

The authors would like to thank Dr. K. Suzuki for technical advice.

(Received September 4, 2011 ; Accepted November 24, 2011)

\section{References}

1) S. Takaki, K. Matsumoto, K. Suzuki ; Appl. Surf. Soc., 33/34, 919 (1988).

2) P. Nath, R. F. Bunshah, B. M. Masel, O. M. Stuffsud ; Thin Solid Films, 72, 463 (1980).

3) T. Oyama, N. Hashimoto, J. Shimizu, Y. Akao, H. Kojima, K. Aikawa, K. Suzuki ; J. Vac. Sci. Technol., A10, 1682 (1992).

4) K. Suzuki, N. Hasimoto, T. Oyama, J. Shimizu, Y. Akao, H. Kojima ; Thin Solid Films, 226, 104 (1993).

5) S. Takaki, Y. Shigesato, H. Harada, H. Kojima, T. Oyama, T. Haranoh ; SID Dig. Tech. Papers, 21, 76 (1990).

6) Y. Shigesato, S. Takaki, T. Haranoh ; Applied Surface Science, 48/49, 269 (1991).

7) S. Ishibashi, Y. Higuchi, Y. Ota, K. Nakamura ; J. Vac. Sci. Technol., A8, 1403 (1990).

8) T. Yamamoto ; Thin Solid Films, 420-421, 100 (2002).

9) T. Hitosugi, A. Ueda, Y. Fubayashi, Y. Hirose, S. Konuma, T. Shimada, T. Hasegawa ; Jpn. J. Appl. Phys., 46, L86 (2007).

10) P. Nath, R. F. Bunshah ; Thin Solid Films, 69, 63 (1980).

11) Y. Ishida, K. Suzuki ; Reports Res. Lab. Asahi Glass Co.,Ltd., 3019 (1991)

12) J. W. Bae, S. D. Park, N. G. Cho, D. H. Lee, G. U. Yeom ; Jpn. J. Appl. Phys., 41, L999 (2002). 